\title{
Parameter Selection in the Parameter Estimation of Grade Transitions in a Polyethylene Plant
}

\author{
Niklas Andersson ${ }^{1}$, Per-Ola Larsson ${ }^{2}$, Johan Åkesson $^{2}$, Niclas Carlsson $^{3}$, Staffan Skålén ${ }^{3} \&$ Bernt Nilsson ${ }^{1}$ \\ ${ }^{1}$ Dept. of Chemical Engineering, Lund University, Lund, Sweden \\ ${ }^{2}$ Modelon AB, Lund, Sweden \\ ${ }^{3}$ Borealis AB, Stenungsund, Sweden \\ Correspondence: Bernt Nilsson, Dept. of Chemical Engineering, Lund University, Lund, Sweden
}

Received: June 18, 2015 Accepted: July 3, 2015 Online Published: September 21, 2015

doi:10.11114/set.v3i1.887 URL: http://dx.doi.org/10.11114/set.v3i1.887

\begin{abstract}
A polyethylene plant at Borealis $\mathrm{AB}$ is modelled in the Modelica language and considered for parameter estimations at grade transitions. Parameters have been estimated for both the steady-state and the dynamic case using the JModelica.org platform, which offers tools for steady-state parameter estimation and supports simulation with parameter sensitivies. The model contains 31 candidate parameters, giving a huge amount of possible parameter combinations. The best parameter sets have been chosen using a parameter-selection algorithm that identified parameter sets with poor numerical properties. The parameter-selection algorithm reduces the number of parameter sets that is necessary to explore. The steady-state differs from the dynamic case with respect to parameter selection. Validations of the parameter estimations in the dynamic case show a significant reduction in an objective value used to evaluate the quality of the solution from that of the nominal reference, where the nominal parameter values are used.
\end{abstract}

Keywords: parameter estimation, parameter selection, Modelica, grade change, polymerisation

\section{Introduction}

Polyethylene manufacturers face a market that is constantly changing, which creates the need to switch between the manufacture of different grades in a cost efficient manner by manipulating the feed of raw materials to the reactors. An existing Borstar ${ }^{\circledR}$ polyethylene plant at Borealis $\mathrm{AB}$ that produces bimodal polyethylene is considered. Bimodal polyethylene products are polymerised in three cascaded reactors: pre-polymerisation, loop and gas-phase reactors (GPR), see Figure 1. The first and smallest reactor is the pre-polymerisation reactor, whose main purpose is to gently polymerise the surface of the catalyst particles. This is necessary since a rapid reaction may damage the particles. The first peak of the bimodal molecular weight distribution is formed in the subsequent loop reactor. The final reactor in the chain, the GPR, is a fluidised bed reactor in which the second peak is formed.

Accurate modelling of advanced chemical reactors is a difficult task, but it is an invaluable tool in cutting the expense of raw materials. Models of chemical processes must be accurately calibrated, in order to make the differences between the model and the real process dynamics as small as possible. We have calibrated a model in order to obtain valid parameters that can be used in a model suitable for optimisation of grade changes, which has previously shown promising results in Larsson (2011). We present here parameter estimation using a differential algebraic equation model (DAE) based on real experimental data responses from the plant, for two different grade changes at two different time periods. We present also cross validation of the results from the parameter estimation.

Polyethylene is formed by a polymerization reaction of ethylene and hydrogen, and its properties depend strongly on its molecular weight. The hydrogen is the termination gas and the ratio between the concentrations of hydrogen and ethylene affects directly the length of the molecular chains formed. Comonomers, such as hexene and buthylene, can be added to control such properties as the density and melting properties. The reaction is catalysed by a Ziegler-Natta catalyst, which makes it possible to operate at low temperatures and pressures. Bimodal polyethylene is the term used to describe polyethylene that has a molecular weight distribution with two peaks. Such a polymer has good resistance and mechanical properties. 


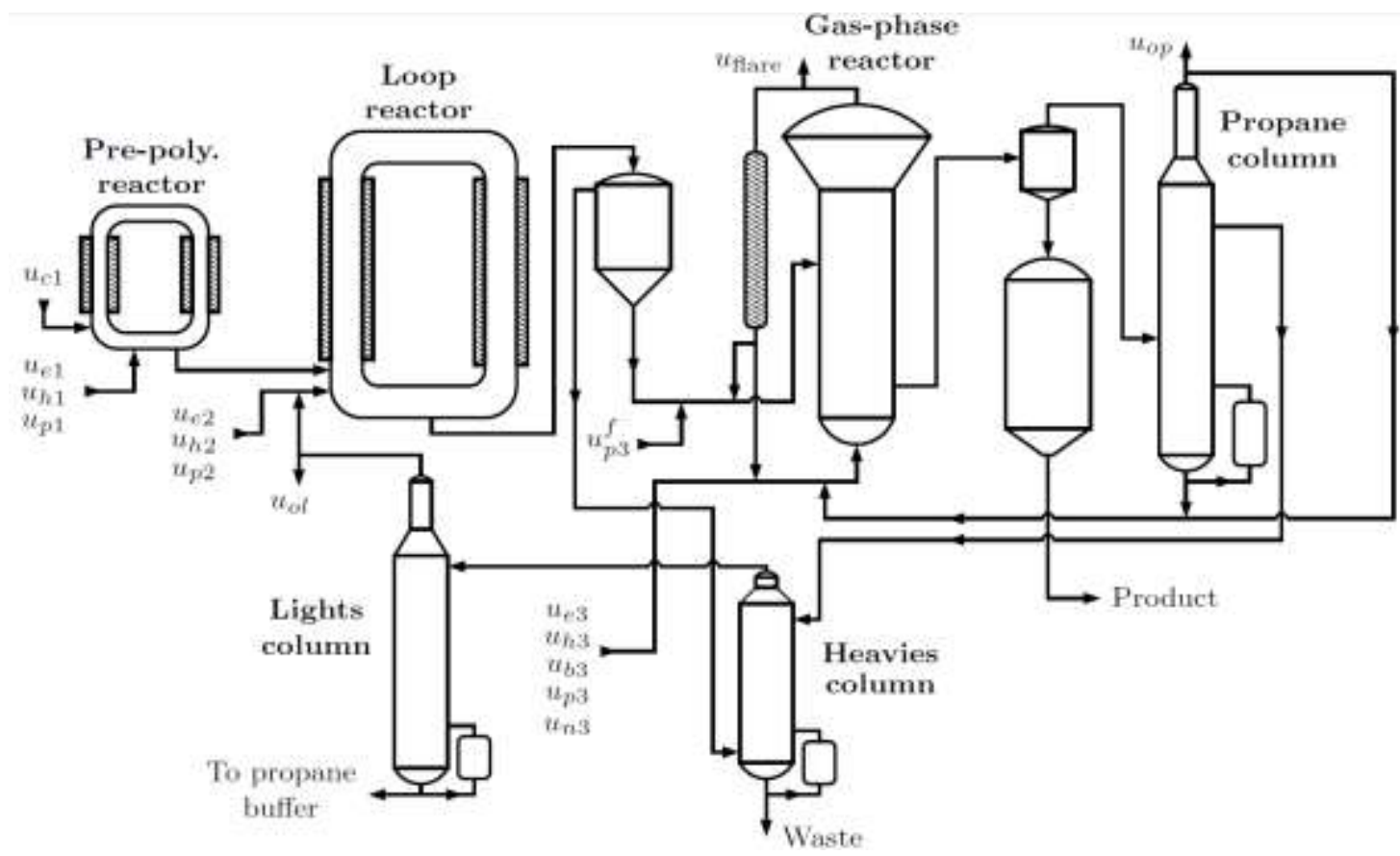

Figure 1. Schematic diagram of the plant PE3 at Borealis AB with the three cascaded Borealis Borstar ${ }^{\circledR}$ reactors and a recycling area with three distillation columns.

Large-scale parameter estimation using non-linear programs (NLP) has previously been successful in many cases. Zavala and Biegler (2006) describe the parameter estimation of a low-density polyethylene tubular reactor at steady state, developing large-scale NLPs that were solved by interior point methods. Sirohi and Choi (1996) presented a parameter estimation of the catalyst parameters of a continuous olefin polymerisation reactor. Faber et al. (2003) calibrated multiple datasets simultaneously by dividing the problem into sub-NLPs. Arora and Biegler (2004) applied a non-linear trust-region successive quadratic programming (SQP) method to estimate the model parameters on-line of a polymerisation reactor model. Another study modelled the high-density polyethylene slurry process where kinetic parameters were estimated (Zhang et al., 2014). They proposed a methodology to transform ill-conditioned parameter estimation problems into well-conditioned subproblems.

The model before parameter estimation is known as the nominal model, and is calibrated in an ad hoc manner using process know-how, results from experiments and/or trial and error. This is satisfactory for a model that is to be used as a predictive controller to correct any discrepancies between the actual and the desired output measurements by updating states or parameters. However, no corrector is available when optimising grade changes offline, and errors in the model will be immediately penalised as the process takes unrealistic paths. It is necessary in this case to calculate the optimal parameters.

Andersson et al. (2011) describe the estimation of parameters at steady state. These parameters were estimated using objective functions in which not only one grade was considered, but also two grades simultaneously. The same model is used in Larsson et al. (2012a) where a model-based optimisation using orthogonal collocation are solved. The process uses a cost function to maximise the profit during grade changes, and uses intervals in grade variables to define on-grade polymer production.

Estimating many parameters simultaneously leads to ill-conditioned problems due to correlations between parameters, and this also reduces the accuracy of the confidence regions. Selecting the best parameters to estimate is therefore important and have been viewed in several studies (Weijers and Vanrolleghem, 1997, Brun et al., 2002 McLean and McAuley, 2012). A good overview of regularization techniques to avoid ill-posed problems are found in Kravaris et al. (2013) where they are divided in heuristic methods and optimization-based methods. The latter category creates combinatorial problems which can be very computationally heavy. An example is a parameter-selection algorithm proposed by Cintrón et al. (2009) that ranked the parameters by two properties, $\alpha$ and $\kappa$. The parameter $\alpha$ quantifies the size of the scaled confidence regions for the parameter set and quantifies how well-conditioned the parameter Jacobian for a parameter set is.

The present paper initially introduces a parameter-reduction scheme, and shows how parameter reduction is performed and validated using a computer cluster. It subsequently investigates an authentic case of a complex model with 
relatively many parameters, showing how a parameter selection algorithm can be successfully used. Finally, it describes how a Modelica library of the components in the polyethylene plant was constructed and used in parameter estimations. It shows also how a Gauss-Newton algorithm can be used together with the Modelica extension Optimica to simulate sensitivities within the platform JModelica.org.

The paper is organised as follows: Section 2 presents an overview of DAE systems, the Newton method and parameter-selection algorithms. Section 3 then presents the tools, methods and models used in the present project. Section 4 describes the parameter-reduction methodology together with the problems used in the parameter-estimation process. Section 5, presents the results, while Section 6 summarises the paper and draws some conclusions.

\section{Theory}

\subsection{Differential Algebraic Equation Systems}

The general non-linear index-1 differential algebraic equation (DAE) form is defined by

$$
\begin{aligned}
& \mathbf{0}=\boldsymbol{F}(\dot{\boldsymbol{x}}, \boldsymbol{x}, \boldsymbol{w}, \boldsymbol{u}, \boldsymbol{p}) \\
& \boldsymbol{y}_{z}=\boldsymbol{g}_{z}(\boldsymbol{x}, \boldsymbol{w}, \boldsymbol{u}, \boldsymbol{p}) \\
& \boldsymbol{y}_{\boldsymbol{u}}=\boldsymbol{g}_{\boldsymbol{u}}(\boldsymbol{x}, \boldsymbol{w}, \boldsymbol{u}, \boldsymbol{p}) \\
& \boldsymbol{x}\left(t_{0}\right)=\boldsymbol{x}_{\mathbf{0}}
\end{aligned}
$$

where $\boldsymbol{x}$ and $\boldsymbol{w}$ are vectors denoting state and algebraic variables, $\boldsymbol{u}$ describe the inputs of the model and $\boldsymbol{p}$ are the parameters of the model treated as constants. The output variables includes both inputs and differential algebraic variables and these are denoted $\boldsymbol{y}_{\boldsymbol{z}}$ and $\boldsymbol{y}_{\boldsymbol{u}}$. Here, $\boldsymbol{z}$ refers to both the algebraic and state variables, that is $\boldsymbol{z}=$ $[\boldsymbol{x}, \boldsymbol{w}]^{T}$. The initial state is defined by $\boldsymbol{x}_{\mathbf{0}}$. The state derivatives are set to 0 when solving the steady-state problem.

\subsection{Non-linear Regression Methods for Differential Algebraic Models}

Regression methods are roughly classified into two broad categories: gradient methods and direct-search methods (Edgar and Himmelblau, 1988). The former depends on accurate parameter gradients, while the latter does not. Gradient methods include the Gauss-Newton Method, the steepest descent method and the Levenberg-Marquardt method, while direct-search include the simplex method (Englezos and Kalogerakis, 2000). Bard 1970 claims that the Gauss-Newton method gives the best results when gradients are available.

The Gauss-Newton method (Englezos and Kalogerakis, 2000) can be used to solve the problems of estimating dynamic parameters, where the Newton step is defined by

$$
\Delta \boldsymbol{p}=\left(\boldsymbol{J}^{T} \boldsymbol{W} \boldsymbol{J}\right)^{-1} \boldsymbol{J}^{T} \boldsymbol{W}(\hat{\boldsymbol{y}}-\boldsymbol{y})
$$

where $\boldsymbol{W}$ is a weighting matrix and $\widehat{\boldsymbol{y}}$ is the measurements corresponding to $\boldsymbol{y}=\left[\boldsymbol{y}_{z}, \boldsymbol{y}_{\boldsymbol{u}}\right]^{T}$. The parameter Jacobian

$$
J=\frac{\partial y}{\partial p}
$$

The sensitivity matrix can be obtained during simulations by adding

$$
\frac{\partial J_{F}}{\partial t}=\left(\frac{\partial F^{T}}{\partial z}\right)^{T} J_{F}+\left(\frac{\partial F^{T}}{\partial p}\right)^{T}
$$

derived by differentiating the DAE system in Eq. (1) and where $J$ is a subset of $J_{F}$. The sensitivity matrix at steady state is given by

$$
J=\frac{\partial g}{\partial p}-\frac{\partial g}{\partial z}\left(\frac{\partial F}{\partial z}\right)^{-1} \frac{\partial F}{\partial p} .
$$

where $\boldsymbol{g}=\left[\boldsymbol{g}_{\mathrm{z}}, \boldsymbol{g}_{\boldsymbol{u}}\right]^{T}$.

A single shooting approach can be used to solve problems of estimating dynamic parameters (Vassiliadis, 1993). It starts with a guess of the parameters. The dynamic model is then simulated, and the parameters are updated iteratively by a regression method, such as Gauss-Newton method.

\subsection{Subset Selection Algorithm}

Studying the sensitivity matrix, $\mathbf{J}$, gives information about the numerical properties of a parameter estimation. Cintrón et al. (2009) suggest an algorithm that investigates parameter sets from a nominal operating point, and ranks the parameter sets according to two quantities: the condition number, $\kappa$, and the parameter selection score, $\alpha$. Here, $\kappa$ is defined as the ratio between the largest and smallest singular value of $\mathbf{J}$, and $\alpha$ is defined by $\alpha(\boldsymbol{p})=|v|$, where

$$
v_{i}=s_{p, i} / p_{i} \text {. }
$$


Here, $s_{p, i}$ are the standard errors of the parameters defined as

$$
s_{p}=\sqrt{\operatorname{diag}(\mathrm{X})} \text {, }
$$

are calculated from the covariance matrix

$$
\mathrm{X}=\frac{1}{n_{y}-n_{p}}|\hat{y}-y|^{2}\left(\boldsymbol{J}^{T} \boldsymbol{W} \boldsymbol{J}\right)^{-1} .
$$

The quantities $\kappa$ and $\alpha$ are used to estimate the parameter dependencies and the uncertainties in the parameters. A low value of $\alpha$ shows that the estimated parameters have been accurately determined, while a low value of $\kappa$ shows that the parameter estimation problem are well determined.

\subsection{Statistics}

Confidence regions are calculated to assess the quality of the parameter estimates. A $1-\beta$ confidence interval for estimated parameters $p^{*}$ can be calculated from

$$
p_{i}^{*} \pm s_{p, i} T_{i n v}\left(\beta / 2, \mathrm{n}_{y}-n_{p}\right)
$$

and means that there is a probability of $1-\beta$ that the true parameter lies within the estimated interval. Here, $T_{i n v}$ is the Student's $t$-distribution and $n_{y}$ and $n_{p}$ are the number of measurements and estimated parameters.

\section{Methods and Tools}

\subsection{Modelling Languages and Tools}

The mathematical model has been developed in Modelica (The Modelica Association, 2011), which is a high-level language for encoding complex physical systems, supporting object-oriented concepts such as classes, components and inheritance. It can also encode textbook-style declarative equations. This modelling paradigm has significant advantages over the block-based paradigm that is often used in the context of physical modelling. In particular, acausal modelling systems do not require the user to solve for the derivatives of a mathematical model. Instead, differential and algebraic equations may be mixed, which then typically results in a differential algebraic equation (DAE).

An extension to Modelica, Optimica, strengthens its optimisation capabilities, by adding a small number of constructs, and enabling the user to specify in a convenient manner optimisation problems based on Modelica models. The parameter estimations in this paper have been performed using JModelica.org, which is a Modelica-based open-source platform targeted at dynamic optimisation (Åkesson et al., 2010). JModelica.org uses an interior point algorithm, IPOPT, to estimate parameters at the steady state (Wächter and Biegler, 2006). Further, JModelica.org uses the Assimulo package (Andersson et al., 2012), which interfaces the IDA solver from the Sundials suite (Hindmarsh et al., 2005). This simulation environment enables parameter sensitivities to be simulated internally, using Eq. (4).

\subsection{Parallel Computing Methodology}

A computer cluster was constructed to provide an environment for distributing parallel batch simulations. The platforms supported by the cluster are python, MATLAB and COMSOL. It consists of a server, clients and slaves, communicating with files written at a shared RAM memory. The server is implemented as a script that handle the queue of batch jobs by distributing them to the next available computer. The client is a script used by the cluster user to distribute the working files. The cluster has 48 cores, composed of five 64-bit computer nodes (Intel Core2 Quad core running at 2.33 $\mathrm{GHz}$ and having $2 \mathrm{~GB}$ RAM), five 64-bit computer nodes (Intel Core i5 7504 cores running at $2.67 \mathrm{GHz}$ and having 4.00 GB RAM), one 64-bit computer node (Intel Xeon, 8 cores running at $2.50 \mathrm{GHz}$ and having $8 \mathrm{~GB}$ RAM).

\subsection{Mathematical Plant Model}

Modelling a series of reactors is a task that involves theoretical and experimental challenges. Borealis $\mathrm{AB}$ uses such a model for the Borstar ${ }^{\circledR}$ process, implemented in OnSpot, a non-linear model predictive control (MPC) software package (Saarinen and Andersen, 2003). This is the basis of the model used in this paper.

The plant consists of three reactors and three distillation columns, Figure 1. The reactors are connected in series starting with the pre-polymerisation reactor, followed by the loop reactor and the gas-phase reactor. All reactors are modelled in a similar manner, based on material balances, and therefore only the loop reactor will be described in detail. McAuley et al. (1995) have described similar models of gas-phase polyethylene reactors, while Larsson et al. (2012b) give a more detailed description of the model used here.

Each reactor is modelled by material balances, where the inflows come from fresh and recycle streams and the outflows pass to a subsequent reactor, a bleed, recycling, or a product outlet. It is assumed that the polymer and the fluids are well-mixed and that the temperatures in the reactors are uniform. The material balances in the loop for the fluids, solids and the total mass of fluids and solids are described by 


$$
\begin{gathered}
\dot{m}_{e 2}=u_{e 2}+w_{e 2}^{r e c}+w_{e 1}+w_{e 2}-r_{e 2} \\
\dot{m}_{h 2}=u_{h 2}+w_{h 2}^{r e c}+w_{h 1}-w_{h 1}-w_{h 2}-r_{h 2} \\
\dot{m}_{p 2}=u_{p 2}+w_{p 2}^{r e c}+w_{p 1}-w_{p 2} \\
\dot{m}_{p e 21}=w_{p e 11}-w_{p e 21} \\
\dot{m}_{p e 2}=r_{p e 2}-w_{p e 22} \\
\dot{m}_{2}=w_{c 1}-w_{c 2} \\
m_{f 2}=m_{e 2}+m_{h 2}+m_{p 2} \\
m_{s 2}=m_{p e 21}+m_{p e 2}+m_{c 2}
\end{gathered}
$$

Here, $m_{i 2}$ are masses in the loop, where Table 1 presents the subscripts $i$. Further, $u_{i 2}$ are the fresh inflows, $w_{i l}$ the flows from the pre-polymerisation reactor, $w_{i 2}$ the flows out from the loop reactor, and $r_{i 2}$ the rates of reaction.

Table 1. The subscripts used, $i$ (left column) and $j$ (right column) .

\begin{tabular}{llll}
\hline Flow & Subscript & Subsystem & Subscript \\
\hline Ethylene & $e$ & Pre-poly. reactor & 1 \\
Hydrogen & $h$ & Loop reactor & 2 \\
Butene & $b$ & Gas-phase reactor & 3 \\
Propane & $p$ & Propane column & 4 \\
Nitrogen & $n$ & Heavies column & 5 \\
Polyethylene & $p e$ & Lights column & 6 \\
Incorp. butene & $p b$ & & \\
Catalyst & $c$ & & \\
Fluids & $f$ & & \\
Solids & $s$ & & \\
\hline
\end{tabular}

The reaction rates are modelled using extended Arrhenius expressions

$$
\begin{aligned}
r_{e 2}= & R_{e 2}\left(m_{c 2}, a_{2}, X_{e 2}, X_{h 2}, P_{2}, p_{r e 1}^{2}, p_{r e 2}^{2}, p_{r e 3}^{2}, p_{r e 1}^{A}\right) \exp \left(\frac{p_{r e 2}^{A}}{T_{2}}\right) \\
& r_{h 2}=R_{h 2}\left(m_{c 2}, a_{2}, X_{h 2}, P_{2}, p_{r h}^{2}\right) \exp \left(\frac{p_{r h}^{A}}{T_{2}}\right) \\
& r_{p e 2}=r_{e 2}+r_{h 2} \\
& r_{2}=r_{e 2}+r_{h 2}
\end{aligned}
$$

where the pre-factors $R_{e 2}$ and $R_{h 2}$ depend on the pressure $\left(P_{2}\right)$, on the reactant concentrations $\left(X_{e 2}\right.$ and $\left.X_{h 2}\right)$, on the catalyst amount $\left(m_{c 2}\right)$ and on the catalyst activity $\left(a_{2}\right)$. The total rate of reaction in the loop, $r_{2}$, is the same as the rate of production of polyethylene in the loop $r_{p e 2}$.

The catalyst activity varies throughout the reactor series and is defined by differential equations involving functions $f_{a j}$ and $f_{d j}$ in each reactor

$$
\begin{gathered}
\dot{a_{2}}=f_{a 2}\left(w_{c 1}, m_{c 2}, a_{2}, d_{2}, a_{1}, d_{1}, r_{p e 2}, p_{c 1}^{A}, p_{c 2}^{A}, p_{c 3}^{A}\right) \\
\dot{d}_{2}=f_{d 2}\left(w_{c 1}, m_{c 2}, a_{2}, d_{2}, a_{1}, d_{1}, p_{c 1}^{A}\right.
\end{gathered}
$$

where aj and $\mathrm{dj}$ are the activation and deactivation, respectively, and $\mathrm{p}_{\mathrm{c} 1}^{\mathrm{A}}, \mathrm{p}_{\mathrm{c} 2}^{\mathrm{A}}$ and $\mathrm{p}_{\mathrm{c} 3}^{\mathrm{A}}$ are parameters. The solid density is modelled as a constant value in the loop. This is a valid assumption because no comonomer is present and the density differences between different grades are small. The fluid density is modelled by an empirical relationship, f $\rho 2$, where

$$
\rho_{f 2}=f_{\rho 2}\left(P_{2}, T_{2}, X_{e 2}, X_{h 2}, X_{p 2}\right) .
$$

The density is used to calculate the total volume in the loop, $\mathrm{p}_{\mathrm{V}}^{2}$ as the sum of the volumes of solids and fluids, expressed by

$$
p_{V}^{2, r e f}=\frac{m_{f 2}}{\rho_{f 2}}+\frac{m_{s 2}}{\rho_{s 2}}
$$

The volume in the loop is controlled by a proportional regulator that manipulates the loop outflow such that

$$
q_{2}=\frac{u_{p 2}+u_{e 2}+u_{h 2}}{\rho_{f 2}}+\frac{w_{p 2}^{r e c}+w_{e 2}^{r e c}+w_{h 2}^{r e c}}{\rho_{f 2}}
$$




$$
+\frac{w_{s 1}}{\rho_{s 2}}+\frac{w_{f 1}}{\rho_{f 2}}-r_{p e 2}\left(\frac{1}{\rho_{f 2}}-\frac{1}{\rho_{s 2}}\right)+K_{2}\left(p_{V}^{2}-p_{V}^{2, r e f}\right),
$$

which is the sum of solid and fluid flows

$$
q_{2}=\frac{w_{s 2}}{\rho_{s 2}}+\frac{w_{f 2}}{\rho_{f 2}}
$$

The loop reactor has settling legs, whose purpose is to yield a higher concentration of solids at the outlet. These legs are modelled with a settling legs factor, s2 $>1$. The ratio between the concentrations of outlet fluid and solid at the outlet is given by

$$
g_{2}=\frac{w_{f 2}}{w_{s 2}}=\frac{1-s_{2} z_{s 2}}{s_{2} z_{s 2}}
$$

where zs 2 is the ratio between the mass of solids and the mass of fluid, and where $\mathrm{s} 2$ is given by fsettl as

$$
s_{2}=f_{\text {settl }}\left(r_{2}, p_{s 1}^{2}, p_{s 2}^{2}, p_{s 3}^{2}\right)
$$

The flows of solids and fluid can now be calculated by combining Equation (27) and (28). In effect of the settling legs in the pre-polymerisation reactor are negligible and the factor is in this case equal to unity. Further, the gas-phase reactor does not have settling legs, and the concentration at the outlet is the same as it is in the reactor.

The instantaneous split factors are defined as the fractions of a polymer that are produced in a certain reactor and are calculated from

$$
\begin{aligned}
& S_{1}=\frac{m_{p e 31}}{m_{p e 31}+m_{p e 32}+m_{p e 3}+m_{p b 3}} \\
& S_{2}=\frac{m_{p e 32}}{m_{p e 31}+m_{p e 32}+m_{p e 3}+m_{p b 3}} \\
& S_{3}=\frac{m_{p e 3}+m_{p b 3}}{m_{p e 31}+m_{p e 32}+m_{p e 3}+m_{p b 3}}
\end{aligned}
$$

where $m_{p e 31}, m_{p e 32}$ and $m_{p e 3}$ are the masses of polymer in the GPR that have been formed in the pre-polymerisation reactor, the loop reactor and the GPR, respectively.

The recycling part of the plant model consists of three distillation towers. The low number of sensors available and the poor quality of the measurements mean that the structure of the model must be simplified. The recycling part is difficult to calibrate also due to the presence of an overlaying control system. A first-order system is therefore used with constant split factors

$$
\begin{aligned}
\dot{w}_{i 4} T_{i 4} & =-w_{i 4}+w_{i 3}, \quad i \in\{e, h, b, p, n\} \\
w_{i 4}^{t} & =S_{i 4}^{t} w_{i 4} \\
w_{i 4}^{s} & =S_{i 4}^{s} w_{i 4} \\
w_{i 4}^{b} & =S_{i 4}^{b} w_{i 4} \\
w_{i 4} & =w_{i 4}^{t}+w_{i 4}^{s}+w_{i 4}^{b}
\end{aligned}
$$

where $T_{i 4}$ is the time constant, $w_{i 4}^{t}, w_{i 4}^{s}, w_{i 4}^{b}$ are the flows to the top, side and bottom, respectively, and $S_{i 4}^{t}, S_{i 4}^{s}, S_{i 4}^{b}$ are the split factor for the top, side and bottom, respectively. The flows $w_{i 4}$ and $w_{i 3}$ are the total outgoing flow from the propane column and from the gas-phase reactor, respectively. The other distillation columns are modelled in the same way.

The model can be expressed as a DAE system with $n_{x}=46, n_{w}=330, n_{u}=12$ and $n_{p}=30$. There are 31 parameters, presented in Table 2, that can be used when calibrating the model, using the indexing system described in Table 1. here are three volume parameters, $p_{V}^{1}, p_{V}^{2}$ and $p_{V}^{3}$, eleven reaction rate parameters for ethylene, $p_{r e}^{1}, p_{r e 1}^{2}, p_{r e 2}^{2}, p_{r e 3}^{2}$, $p_{r e 1}^{3}, p_{r e 2}^{3}, p_{r e 3}^{3}, p_{r e 4}^{3}, p_{r e 5}^{3}, p_{r e 1}^{A}$ and $p_{r e 2}^{3}$, three reaction parameters for butylene, $p_{r b 1}^{3}, p_{r b 2}^{3}$ and $p_{r b 3}^{3}$, four kinetic reaction parameters for hydrogen, $p_{r h}^{1}, p_{r h}^{2}, p_{r h}^{3}$ and $p_{r h}^{A}$, three catalyst activity parameters $p_{c 1}^{A}, p_{c 2}^{A}$ and $p_{c 3}^{A}$, three settling legs parameters $p_{s 1}^{2}, p_{s 2}^{2}$ and $p_{s 3}^{2}$, one parameter (which is the reference value) for the bed level in the gas-phase reactor, $p_{\text {bed }}^{3}$, and two offset parameters for nitrogen and propylene, $p_{o n}^{3}$ and $p_{o p}^{3}$. Most parameters are kinetic parameters, describing the rates of reactions and of catalyst deactivations, although some parameters that affect flows, pressures and levels must also be calibrated. Calibrating all parameters in the parameter-estimation problem simultaneously leads to poorly estimated parameters with high uncertainties. An algorithm known as the subset selection algorithm (SSA) has been used to identify the most appropriate parameters (Cintrón et al., 2009). 
Table 2. The parameters selected for use in the subset selection algorithm.

\begin{tabular}{cccc}
\hline Prepoly & Loop & GPR & All \\
\hline$p_{V}^{1}$ & $p_{V}^{2}$ & $p_{V}^{3}$ & $p_{r e 1}^{A}$ \\
$p_{r e}^{1}$ & $p_{s 1}^{2}$ & $p_{b e d}^{3}$ & $p_{r e 2}^{A}$ \\
$p_{r h}^{1}$ & $p_{s 2}^{2}$ & $p_{\text {rh }}^{3}$ & $p_{r h}^{A}$ \\
& $p_{s 3}^{2}$ & $p_{r e 2}^{3}$ & $p_{c 1}^{A}$ \\
& $p_{r e 1}^{2}$ & $p_{r e 3}^{3}$ & $p_{c 2}^{A}$ \\
& $p_{r e 2}^{2}$ & $p_{r e 4}^{3}$ & $p_{c 3}^{A}$ \\
& $p_{r e 3}^{2}$ & $p_{r e 5}^{3}$ & \\
& $p_{r h}^{2}$ & $p_{r e 1}^{3}$ & \\
& $p_{r e}^{2}$ & $p_{r b 1}^{3}$ & \\
& & $p_{o n}^{3}$ & \\
& & $p_{o p}^{3}$ & \\
& & $p_{r b 2}^{3}$ & \\
& & $p_{r b 3}^{3}$ & \\
& &
\end{tabular}

The model contains 12 inputs, $\mathbf{u}$, comprising $u_{e l}, u_{h 1}, u_{p 1}$ and $u_{c l}$ for the pre-polymerisation reactor, $u_{e 2}, u_{h 2}$ and $u_{p 2}$ for the loop reactor, and $u_{e 3}, u_{h 3}, u_{b 3}, u_{p 3}$ and $u_{n 3}$ for the GPR. The outputs $\mathbf{y}_{\mathbf{u}}$ are the same signals as the inputs $\mathbf{u}$. The outputs $\mathbf{y}_{\mathbf{z}}$ include 14 signals, where $m_{s 2}$ is the mass of solids in the loop reactor, $m_{e 3}, m_{p 3}, m_{s 3}$ are the masses of ethylene, propylene and solids in the GPR, $x_{h 2}, x_{e 2}$ are the mass concentrations of hydrogen and ethylene in the loop reactor, $x_{h 3}, x_{n 3}, x_{b 3}, x_{e 3}$ are the mass concentrations of hydrogen, nitrogen, butylene and ethylene in the GPR, $r_{2}$ and $r_{3}$ are the total reaction rates in the loop reactor and GPR, $S_{3}$, is the instantaneous split factor for the GPR and, finally, $P_{3}$, is the pressure in the GPR.

\subsection{Data processing}

Signals are collected from measurements in the plant and are downsampled and filtered using a low-pass Butterworth filter with a frequency chosen by considering the Nyquist-Shannon sampling theorem.

\section{Parameter Estimation Methodology for Large-scale Systems}

The number of parameter combinations increases rapidly with the number of parameters and a parameter-selection method must be used to reduce the number of parameter sets. The selection method consists of two loops, the SSA loop and the parameter estimation loop, each one consisting of three base parts: combination, evaluation and filter blocks, Figure 2. The blocks are defined as follows:

Combination is the process of taking an input population $P_{i n}=\left\{P_{i n}^{1}, \ldots, P_{i n}^{n_{p i n}}\right\}$ with $n_{\text {pin }}$ parameter sets and mixing it with all $n_{P 0}$ parameters $P_{0}=\left\{p_{1}, \ldots, p_{n_{P 0}}\right\}$ to create a new parameter set population $P_{\text {out }}$ that contains parameter sets with one more parameter than the parameter sets of the input population. The input population is empty before the first iteration, and thus the output population will contain one parameter set for every parameter in $P_{0} . P_{\text {in }}$ is not empty before the next iteration, and thus the $P_{i n}^{1}$ will enter the output population as $n_{P 0}$ parameter sets defined by $\left\{\left\{P_{i n}^{1}, p_{1}\right\}, \ldots,\left\{P_{i n}^{1}, p_{n_{P 0}}\right\}\right\}$, and $P_{i n}^{2}, \ldots, P_{i n}^{n_{p i n}}$ will be combined in the same way. The same parameter set can be created from two different parameter sets in the input population, and thus an operation is carried out to remove all duplicates. The maximum number of parameter sets in the output population is $n_{P 0} n_{p i n}$, but this may be reduced when duplicates are removed. There are two combination blocks, Block 1 in the SSA loop and Block 4 in the parameter estimation loop.

SSA Evaluation evaluates $\alpha$ and $\kappa$ values for each parameter set of the input population as defined in Section 2.3, and calculates a score $\theta$, given by $\theta=\lg \alpha+\lg \kappa$, that is later used in the filter block to determine the best parameter sets in the SSA loop.

Parameter estimation is the step where parameter estimations are made for all parameter sets in the input population, and is evaluated in the cluster. All parameter sets are passed as batch jobs to the cluster and an objective value that measures the deviation between model and measurements is returned. The parameter estimation step is the most computationally expensive step.

Filters are used to reduce the number of parameter sets, which otherwise would increase rapidly. There are two filter blocks, one in each loop. The filter block takes a population of parameter sets, a score that has been calculated to rank the parameter sets, and a cutoff that defines how many parameter sets should pass. In the first loop, $\theta$ is used as score and $n_{S S A}$ is used as cutoff. In the second loop, $Q$ is used as score and $n_{Q}$ is used as cutoff. The cutoffs have been chosen to $n_{S S A}=300$ and $n_{Q}=2$ in the work presented here to limit the number of calculations.

SSA evaluation is relatively cheap, but the number of parameter sets increases rapidly as $\mathrm{n}$ par increases. The number of parameter sets increases as the binomial coefficients $\left(\begin{array}{c}N_{p} \\ k\end{array}\right)$, which for $n_{p}=31$ are $\{31,465,4495,31465,169911, \ldots\}$. Setting a filter cutoff limits the population that must be examined to $n_{\text {cutoff }} n_{P O}$ instead. 


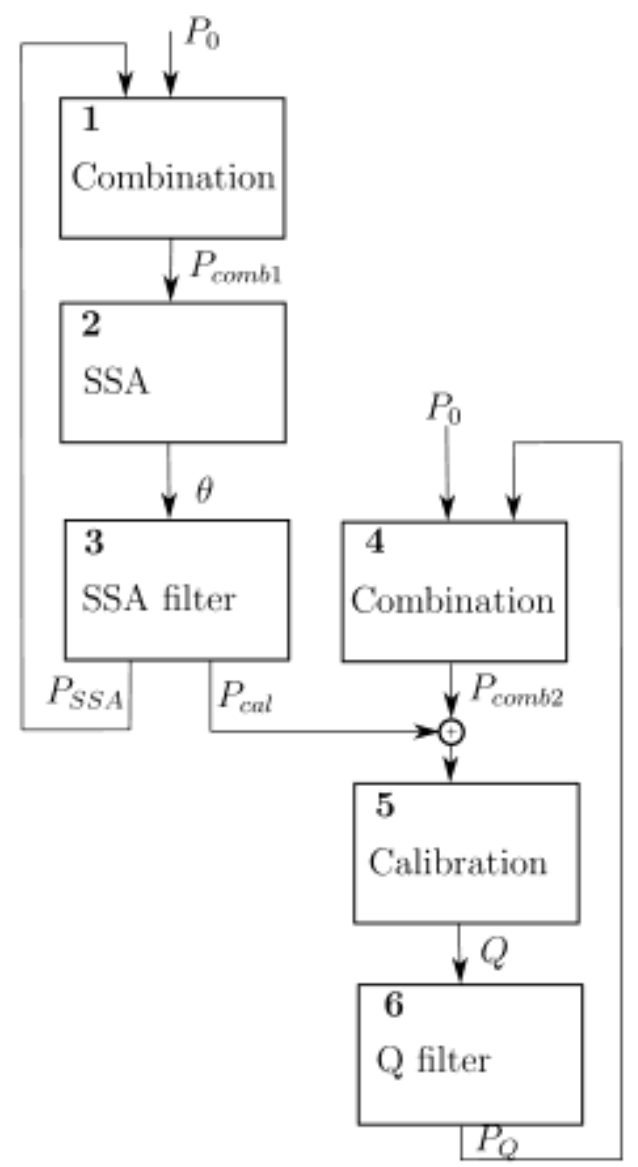

Figure 2. The SSA selection procedure used.

\subsection{Parameter Estimation Procedure}

Parameter estimations are investigated for both the steady-state case and the dynamic case. Both the starting grade and the final grade are calibrated simultaneously when considering steady-state parameter estimation, to make it easier to compare the results to those obtained from the dynamic parameter estimation, where the system is simulated from the starting grade to the final grade.

\subsubsection{Steady-state Parameter Estimation}

For both the starting grade and the end grade, the output signals $\boldsymbol{y}_{\boldsymbol{z}}$ and $\boldsymbol{y}_{\boldsymbol{u}}$ are averaged to give $\widehat{\boldsymbol{y}}_{\boldsymbol{z}}$ and $\widehat{\boldsymbol{y}}_{\boldsymbol{u}}$. Table 3 lists scaled measurements, $\widehat{\boldsymbol{y}}$, and their standard deviations, $\sigma$, for some of the inputs and outputs. The deviation between the measured signal and the signal obtained from the model can be expressed as an objective function of weighted least squares for the differential algebraic variables

$$
Q_{z}=\sum_{i=\{\text { grades }\}}\left(\widehat{\boldsymbol{y}}_{z, \boldsymbol{i}}-\boldsymbol{y}_{\boldsymbol{z}, \boldsymbol{i}}\right) \boldsymbol{W}\left(\boldsymbol{y}_{z, \boldsymbol{i}}\right)\left(\widehat{\boldsymbol{y}}_{\boldsymbol{z}, \boldsymbol{i}}-\boldsymbol{y}_{z, \boldsymbol{i}}\right),
$$

Where $\boldsymbol{W}\left(\boldsymbol{y}_{z, i}\right)$ is the diagonal weighting matrix

$$
\boldsymbol{W}\left(\boldsymbol{y}_{z, i}\right)=\left(\begin{array}{cccc}
\frac{1}{\hat{y}_{z, 1}^{2}} & 0 & \cdots & 0 \\
0 & \frac{1}{\hat{y}_{z, 2}^{2}} & \ddots & 0 \\
\vdots & \ddots & \ddots & \vdots \\
0 & 0 & \cdots & \frac{1}{\hat{y}_{z, n}^{2}}
\end{array}\right),
$$

For $i=1, \ldots, N_{z}$. The deviation for the inputs can be expressed by

$$
Q_{u}=\sum_{i=\{\text { grades }\}}\left(\widehat{\boldsymbol{y}}_{\boldsymbol{u}, \boldsymbol{i}}-\boldsymbol{y}_{\boldsymbol{u}, \boldsymbol{i}}\right) \boldsymbol{W}\left(\boldsymbol{y}_{\boldsymbol{u}, \boldsymbol{i}}\right)\left(\widehat{\boldsymbol{y}}_{\boldsymbol{u}, \boldsymbol{i}}-\boldsymbol{y}_{\boldsymbol{u}, \boldsymbol{i}}\right),
$$

where $\boldsymbol{W}\left(\boldsymbol{y}_{u, i}\right)$ is the corresponding weighting matrix for the inputs.

The steady-state parameter estimation of the system (Eq. (1)) is formulated as an optimisation problem

$$
\min _{\boldsymbol{p}, \boldsymbol{u}} Q_{z}+Q_{u}
$$




$$
\begin{array}{ll}
\text { subj. to } & \boldsymbol{F}(\dot{\boldsymbol{x}}, \boldsymbol{x}, \boldsymbol{w}, \boldsymbol{p}, \boldsymbol{u})=0 \\
& \dot{\boldsymbol{x}}=0 \\
& \boldsymbol{y}_{z}=\boldsymbol{g}_{z}(\boldsymbol{z}) \\
& \boldsymbol{y}_{\boldsymbol{u}}=\boldsymbol{g}_{\boldsymbol{u}}(\boldsymbol{u}) \\
& \boldsymbol{x}_{\text {min }} \leq \boldsymbol{x} \leq \boldsymbol{x}_{\text {max }} \\
& \boldsymbol{w}_{\text {min }} \leq \boldsymbol{w} \leq \boldsymbol{w}_{\text {max }} \\
& \boldsymbol{u}_{\text {min }} \leq \boldsymbol{u} \leq \boldsymbol{u}_{\text {max }}
\end{array}
$$

Both the starting grade and end grade are included in the steady-state parameter estimation, and this means that it is necessary to calibrate $N_{u}$ inflows for the starting grade and $N_{u}$ inflows for the end grade. Further, $N_{p}$ parameters must be calibrated and the number of degrees of freedom is thus $2 N_{u}+N_{p}$.

\subsubsection{Dynamic Parameter Estimation}

The dynamic parameter estimation procedure is solved by a single shooting procedure. The initial states are found by solving the steady-state parameter estimation problem, defined in Equation (41) for the starting grade only, with the parameter values that have been obtained being set as constants. The system is subsequently simulated, with the inputs $\mathbf{u}$ following the measurement data, $\widehat{\boldsymbol{y}}_{\boldsymbol{u}}$, from the starting grade that has been achieved to the end grade. The parameters can be updated by minimising the objective value defined as the weighted sum of squares of the residuals

$$
S(\mathbf{p})=\sum_{i=1}^{N_{t}}\left(\widehat{\boldsymbol{y}}_{\boldsymbol{z}, \boldsymbol{i}}-\boldsymbol{y}_{\boldsymbol{z}}\left(t_{i}, \mathbf{p}\right)\right) \boldsymbol{W}\left(\boldsymbol{y}_{\boldsymbol{z}, \boldsymbol{i}}\right)\left(\widehat{\boldsymbol{y}}_{\boldsymbol{z}, \boldsymbol{i}}-\boldsymbol{y}_{\boldsymbol{z}}\left(t_{i}, \mathbf{p}\right)\right)
$$

where $N_{t}$ is the number of time points and $\boldsymbol{y}_{z}\left(t_{i}, \mathbf{p}\right)$ is the model outputs from the simulation at time $t_{i}$. The simulation follows the inputs $\widehat{\boldsymbol{y}}_{\boldsymbol{u}}$, and thus only the outputs $\boldsymbol{y}_{\boldsymbol{z}}\left(t_{i}, \mathbf{p}\right)$ need to be included in the objective value. The parameter estimation problem is solved iteratively using the Gauss-Newton algorithm, described in Section 2.2, to update the parameters.

\section{Parameter Estimation Results}

A grade change is the process in which a plant producing Grade $G_{1}$ starts to produce Grade $G_{2}$. Two datasets for two different grades $\left(T_{1}\right.$ and $\left.T_{2}\right)$ are analysed. The four datasets are denoted $T_{1}^{1}, T_{1}^{2}, T_{2}^{1}$ and $T_{2}^{2}$. Measurements are available for 26 signals in the objective function, where 12 signals are raw material inflows and 14 signals are measured states.

\subsection{Steady-state Parameter Estimation}

Figure 3 presents the results from the SSA analysis. The lowest values of $\alpha$ and $\kappa$ remain quite constant as more parameters are added. The parameter sets with best objective values are marked with a triangle and are located in the lower left corners for parameter sets having between two and five parameters, and at relatively large values of $\alpha$ and $\kappa$ for parameter sets having six or seven parameters. The parameters for parameter sets having six or seven parameters are not located with the SSA loop of the algorithm, but they are located within the parameter estimation loop, marked in green, that has been created by parameter combinations from the best objective values in the preceding iteration. These parameter sets, however, have very poor numerical properties, $\alpha=10^{4.09}$ and $\kappa=10^{9.49}$. The $2 N_{u}$ input parameters are also calibrated and included in $\mathbf{J}$ in the steady-state case, which makes the problem harder to solve. 

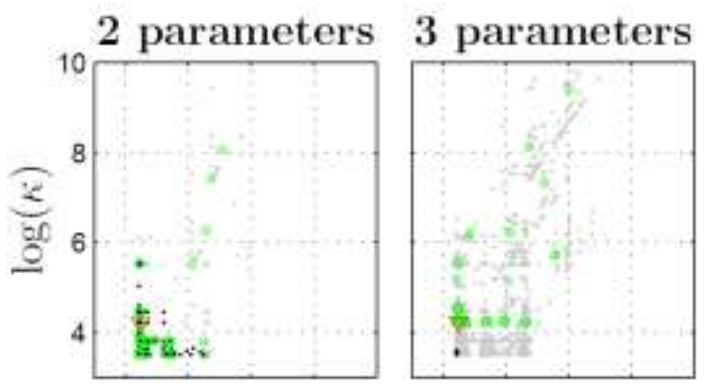

4 parameters
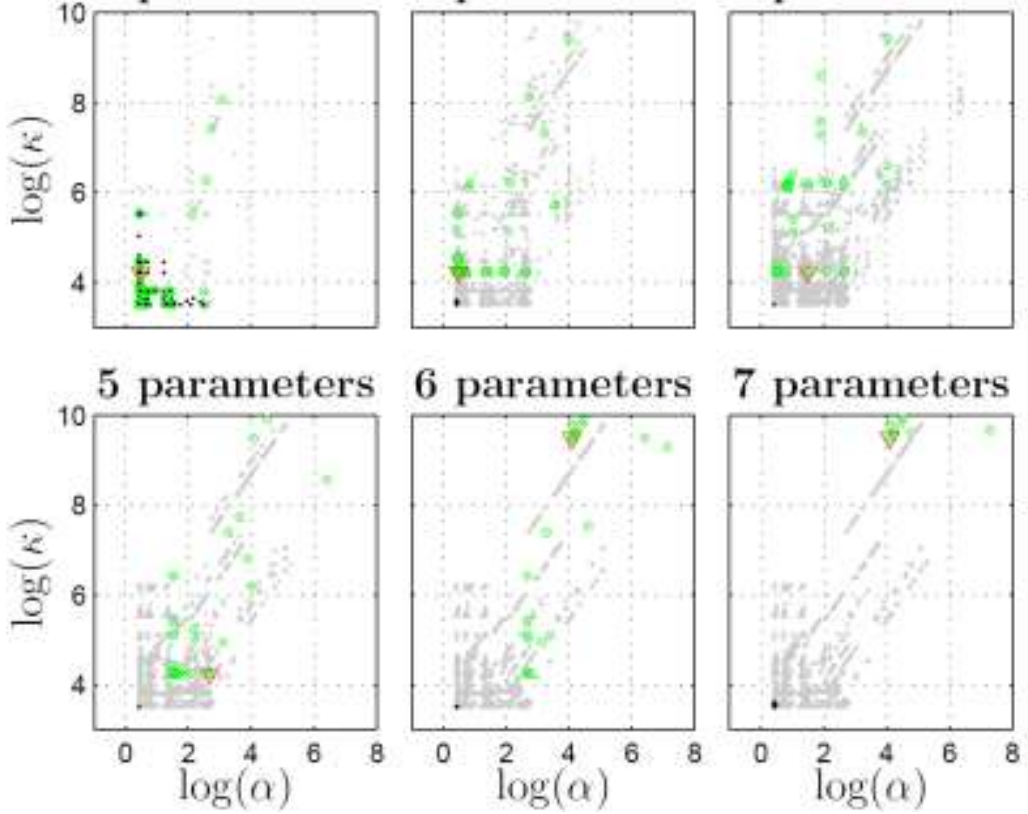

Figure 3. SSA evaluations for the steady-state case for two to seven number of parameters. The parameter sets are marked in gray, the best parameters from the SSA loop are marked in black, and the parameters from the parameter estimation loop are marked in green. The parameter sets with the best objective values are marked with red triangles. 
Table 3. Measurements $(\hat{y})$ and standard deviations $(\sigma)$ for all datasets together with the calibrated model outputs $y^{*}$ at steady-state and $95 \%$ confidence interval for all inputs $y_{u}$. All values have been scaled to the measurements of the starting grade of dataset $T_{1}^{1}$.

\begin{tabular}{|c|c|c|c|c|c|c|c|c|c|}
\hline & $G_{1}^{1}$ & & $G_{1}^{2}$ & & & $G_{1}^{1}$ & & $G_{1}^{2}$ & \\
\hline & $\begin{array}{l}\hat{y}_{u} \\
(\sigma)\end{array}$ & $\begin{array}{l}y_{u}^{*} \pm \\
\text { conf. }\end{array}$ & $\begin{array}{l}\hat{y}_{u} \\
(\sigma)\end{array}$ & $\begin{array}{l}y_{u}^{*} \pm \\
\text { conf. }\end{array}$ & & $\begin{array}{l}\hat{y}_{z} \\
(\sigma)\end{array}$ & $y_{z}^{*}$ & $\begin{array}{l}\hat{y}_{z} \\
(\sigma)\end{array}$ & $y_{z}^{*}$ \\
\hline$u_{p 1}$ & $\begin{array}{c}1.00 \\
(0.00)\end{array}$ & $\begin{array}{c}0.99 \pm \\
0.00\end{array}$ & $\begin{array}{c}1.00 \\
(0.00)\end{array}$ & $\begin{array}{c}1.01 \pm \\
0.00\end{array}$ & $r_{2}$ & $\begin{array}{c}1.00 \\
(0.00)\end{array}$ & 0.95 & $\begin{array}{c}1.03 \\
(0.00)\end{array}$ & 0.94 \\
\hline$u_{e 1}$ & $\begin{array}{c}1.00 \\
(0.00)\end{array}$ & $\begin{array}{c}1.00 \pm \\
0.00\end{array}$ & $\begin{array}{c}0.99 \\
(0.00)\end{array}$ & $\begin{array}{c}0.98 \pm \\
0.00\end{array}$ & $m_{s 2}$ & $\begin{array}{c}1.00 \\
(0.01)\end{array}$ & 1.10 & $\begin{array}{c}1.07 \\
(0.01)\end{array}$ & 1.18 \\
\hline$u_{h 1}$ & $\begin{array}{c}1.00 \\
(0.00)\end{array}$ & $\begin{array}{c}1.00 \pm \\
4.14\end{array}$ & $\begin{array}{c}0.92 \\
(0.01)\end{array}$ & $\begin{array}{c}0.92 \pm \\
4.14\end{array}$ & $X_{e 2}$ & $\begin{array}{c}1.00 \\
(0.00)\end{array}$ & 1.06 & $\begin{array}{c}1.16 \\
(0.00)\end{array}$ & 1.18 \\
\hline$u_{c 1}$ & $\begin{array}{c}1.00 \\
(0.01)\end{array}$ & $\begin{array}{c}0.99 \pm \\
0.08\end{array}$ & $\begin{array}{c}0.82 \\
(0.01)\end{array}$ & $\begin{array}{c}0.86 \pm \\
0.06\end{array}$ & $X_{h 2}$ & $\begin{array}{c}1.00 \\
(0.03)\end{array}$ & 1.04 & $\begin{array}{c}1.22 \\
(0.01)\end{array}$ & 1.27 \\
\hline$u_{e 2}$ & $\begin{array}{c}1.00 \\
(0.00)\end{array}$ & $\begin{array}{c}0.94 \pm \\
0.00\end{array}$ & $\begin{array}{c}1.04 \\
(0.00)\end{array}$ & $\begin{array}{c}0.94 \pm \\
0.00\end{array}$ & $r_{3}$ & $\begin{array}{c}1.00 \\
(0.00)\end{array}$ & 1.07 & $\begin{array}{c}0.95 \\
(0.00)\end{array}$ & 0.91 \\
\hline$u_{h 2}$ & $\begin{array}{c}1.00 \\
(0.05)\end{array}$ & $\begin{array}{c}0.89 \pm \\
0.21\end{array}$ & $\begin{array}{c}1.05 \\
(0.02)\end{array}$ & $\begin{array}{c}0.96 \pm \\
0.21\end{array}$ & $S_{3}$ & $\begin{array}{c}1.00 \\
(0.00)\end{array}$ & 1.05 & $\begin{array}{c}0.96 \\
(0.00)\end{array}$ & 0.98 \\
\hline$u_{p 2}$ & $\begin{array}{c}1.00 \\
(0.02)\end{array}$ & $\begin{array}{c}0.98 \pm \\
0.00\end{array}$ & $\begin{array}{c}0.78 \\
(0.01)\end{array}$ & $\begin{array}{c}0.79 \pm \\
0.00\end{array}$ & $X_{e 3}$ & $\begin{array}{c}1.00 \\
(0.02)\end{array}$ & 1.01 & $\begin{array}{c}0.82 \\
(0.00)\end{array}$ & 0.85 \\
\hline$u_{p 3}$ & $\begin{array}{c}1.00 \\
(0.42)\end{array}$ & $\begin{array}{c}1.10 \pm \\
0.01\end{array}$ & $\begin{array}{c}0.00 \\
(0.01)\end{array}$ & $\begin{array}{c}0.00 \pm \\
0.01\end{array}$ & $X_{b 3}$ & $\begin{array}{c}1.00 \\
(0.01)\end{array}$ & 0.91 & $\begin{array}{c}0.87 \\
(0.02)\end{array}$ & 0.92 \\
\hline$u_{h 3}$ & $\begin{array}{c}1.00 \\
(0.07)\end{array}$ & $\begin{array}{c}0.84 \pm \\
1.22\end{array}$ & $\begin{array}{c}0.21 \\
(0.02)\end{array}$ & $\begin{array}{c}0.23 \pm \\
0.58\end{array}$ & $X_{h 3}$ & $\begin{array}{c}1.00 \\
(0.02)\end{array}$ & 1.11 & $\begin{array}{c}0.19 \\
(0.00)\end{array}$ & 0.18 \\
\hline$u_{b 3}$ & $\begin{array}{c}1.00 \\
(0.01)\end{array}$ & $\begin{array}{c}1.04 \pm \\
0.00\end{array}$ & $\begin{array}{c}1.03 \\
(0.02)\end{array}$ & $\begin{array}{c}0.97 \pm \\
0.00\end{array}$ & $X_{n 3}$ & $\begin{array}{c}1.00 \\
(0.03)\end{array}$ & 0.79 & $\begin{array}{c}1.04 \\
(0.00)\end{array}$ & 1.21 \\
\hline$u_{e 3}$ & $\begin{array}{c}1.00 \\
(0.01)\end{array}$ & $\begin{array}{c}1.05 \pm \\
0.00\end{array}$ & $\begin{array}{c}0.95 \\
(0.00)\end{array}$ & $\begin{array}{c}0.89 \pm \\
0.00\end{array}$ & $P_{3}$ & $\begin{array}{c}1.00 \\
(0.00)\end{array}$ & 0.98 & $\begin{array}{c}1.00 \\
(0.00)\end{array}$ & 0.97 \\
\hline$u_{n 3}$ & $\begin{array}{c}1.00 \\
(0.28)\end{array}$ & $\begin{array}{c}1.15 \pm \\
0.05\end{array}$ & $\begin{array}{c}3.30 \\
(0.15)\end{array}$ & $\begin{array}{c}1.64 \pm \\
0.05\end{array}$ & $m_{s 3}$ & $\begin{array}{c}1.00 \\
(0.01)\end{array}$ & 1.14 & $\begin{array}{c}1.07 \\
(0.01)\end{array}$ & 1.14 \\
\hline & & & & & $m_{p 3}$ & $\begin{array}{c}1.00 \\
(0.01)\end{array}$ & 1.00 & $\begin{array}{c}1.07 \\
(0.00)\end{array}$ & 0.95 \\
\hline & & & & & $m_{e 3}$ & $\begin{array}{c}1.00 \\
(0.02)\end{array}$ & 0.87 & $\begin{array}{c}0.82 \\
(0.01)\end{array}$ & 0.73 \\
\hline
\end{tabular}


Table 4. Results from the steady-state parameter selection algorithm.

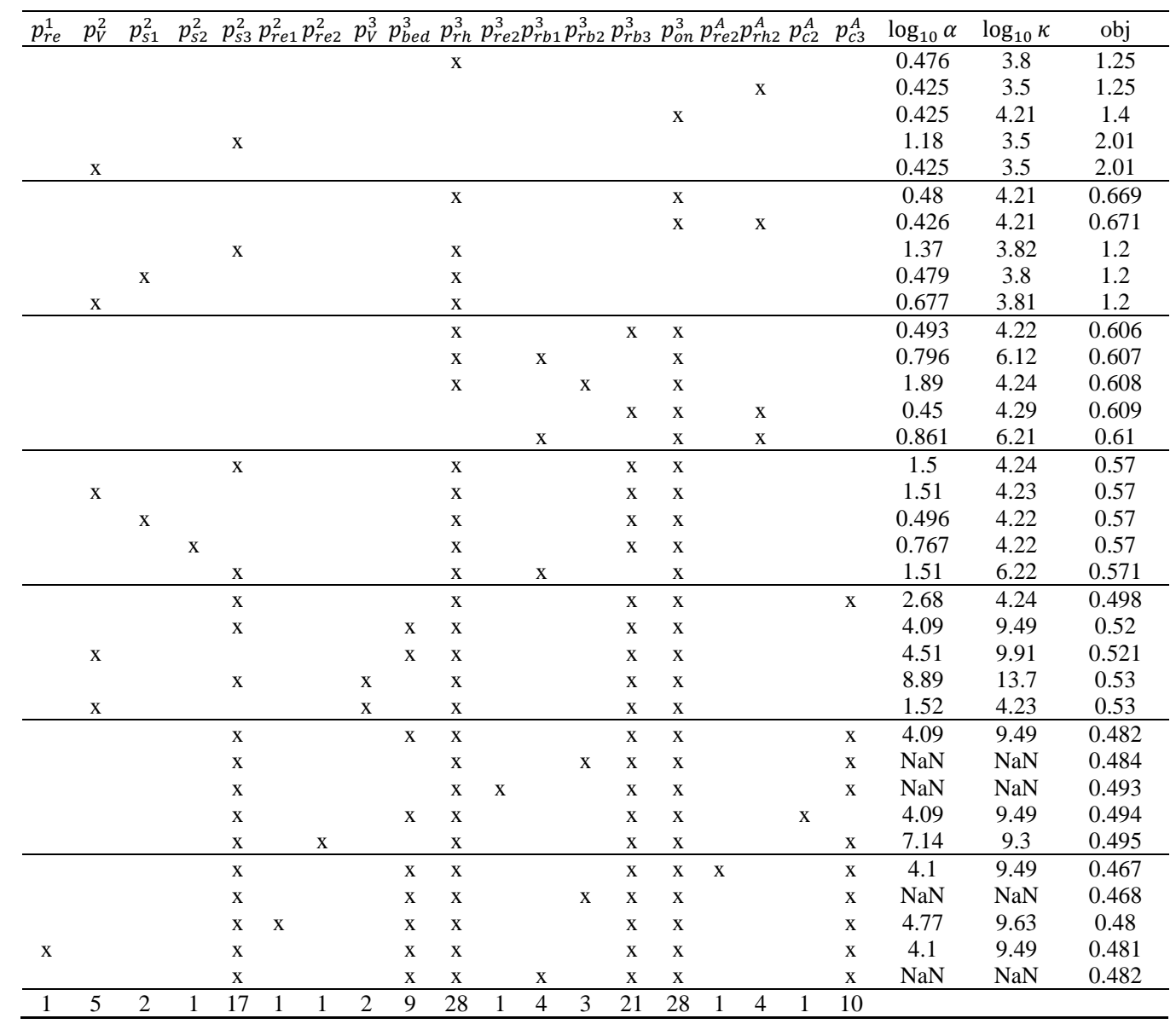

Table 5. Estimated scaled parameters for the diff erent datasets for the best parameter set with five parameters, for the steady-state case.

\begin{tabular}{lllccl}
\hline & $p_{s 3}^{2}$ & \multicolumn{1}{c}{$p_{r h}^{3}$} & $p_{o n}^{3}$ & $p_{c 3}^{A}$ & \multicolumn{1}{c}{$p_{r b 3}^{3}$} \\
\hline$p_{T_{1}^{1}}^{*}$ & 1.78 & 11.21 & 0.314 & 3.11 & 1.37 \\
$p_{T_{1}^{2}}^{*}$ & 1.10 & 6.68 & 0.734 & 0.00 & 1.42 \\
$p_{T_{2}^{1}}^{*}$ & 0.286 & 19.12 & 0.554 & 0.00 & 1.32 \\
$p_{T_{2}^{2}}^{*}$ & 0.841 & 21.332 & 0.00 & 0.892 & 1.526 \\
\hline
\end{tabular}




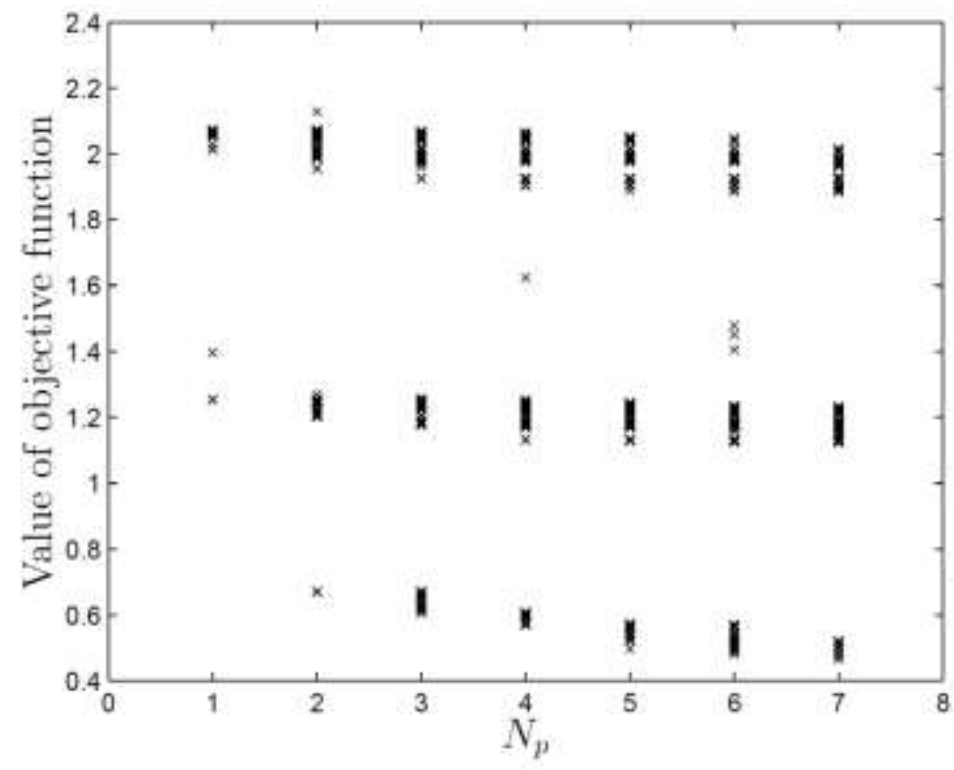

Figure 4. The objective values plotted against the number of parameters for steady-state parameter estimation.

Figure 4 shows the objective value as a function of the number of parameters. The objective function value falls as the number of parameters is increased, eventually reaching a constant value at about five parameters. Adding more parameters than five does not improve the objective value. The objective values are grouped in three distinctive bands, where the lower band contains the two best parameters.

Table 4 shows that the parameter set for five parameters, that has the best objective value is $\left\{p_{s 3}^{2}, p_{r h}^{3}, p_{o n}^{3}, p_{c 3}^{A}, p_{r b 3}^{3}\right\}$. Table 3 shows the values of the signals in the objective function for this parameter estimation. The calibrated values agree with the measurements for most signals, but deviate in, for example, the nitrogen values. This is because both $u_{n 3}$ and $X_{n 3}$ are included in the objective function and it is hard to achieve a good fit for both. The confidence intervals for many variables are very narrow, although they are high for the signals $u_{h 1}$ and $u_{h 3}$. These two signals are the main contribution to the high $\alpha$ values in the steady-state case.

Table 5 shows the parameters for the best parameter set with five parameters. The parameters $p_{s 3}^{2}, p_{r h}^{3}, p_{o n}^{3}, p_{c 3}^{A}$ all vary greatly between the datasets, which is compatible with the high values of $\alpha=10^{2.68}$ and $\kappa=10^{4.24}$. The parameter $p_{r b 3}^{3}$ does not vary very much between the datasets.

The best parameter sets with one parameter are $p_{r h}^{3}$ and $p_{r h}^{A}$, which have almost equal objective values. Both parameters affect principally the hydrogen concentration, where the initial deficiency is largest. These parameters are also included in the two best parameter sets with two parameters, $\left\{p_{r h}^{3}, p_{o n}^{3}\right\}$ and $\left\{p_{o n}^{3}, p_{r h}^{A}\right\}$, and one could expect that the parameter set $\left\{p_{r h}^{3}, p_{o n}^{3}, p_{r h}^{A}\right\}$ is the best set with three parameters. However, this parameter set was not solvable because the parameters $p_{r h}^{3}$ and $p_{r h}^{A}$ both model the hydrogen concentration, and this leads to an ill-conditioned problem. This parameter set is efficiently excluded by the SSA algorithm. The parameter $p_{r h}^{3}$ becomes much more important than $p_{r h}^{A}$ as more parameters are added, and the parameters never occur together.

The objective values are low, while values of $\alpha$ are high, indicating that the validations may be very inaccurate. A possible explanation of the inaccuracy is that the inflow values $y_{u}$ are calibrated together with the parameters, and these may depend on each other.

\subsection{Dynamic Parameter Estimation}

Figure 5 shows the results from a parameter estimation in which four molar fractions $x_{i j}$ and two masses $m_{i j}$ were modelled. The calibrated model response coincides much better with the measured data than it coincides with the nominal model (which has been simulated with nominal parameters). The calibrated and simulated values are not equal, but the profiles follow the same trend. An SSA evaluation of the parameter sets was carried out in order to reduce the number of parameters (Figure 6). The values of $\alpha$ and $\kappa$ are low, as desired, for models with few parameters. The values of $\alpha$ and $\kappa$ increase as the number of parameters increases, and the dot cloud moves upwards and to the right. This shows that the parameter sets become harder to calibrate and the uncertainties become larger. The locations of the best objective values, marked in the figure, show that the best objective value is not correlated with small uncertainty in the parameters, and this is important to consider when choosing a good parameter set. It is interesting to see how many parameters are needed to reach a desired objective value. Therefore, 300 parameter set with the best values of $\alpha$ and $\kappa$ 
were calibrated for each number of parameters, and their objective values were plotted against the number of parameters (Figure 7). As expected, the best objective values decrease as more parameters are added, saturating at five parameters. Adding more parameters than five does not lead to a decrease in the objective value.

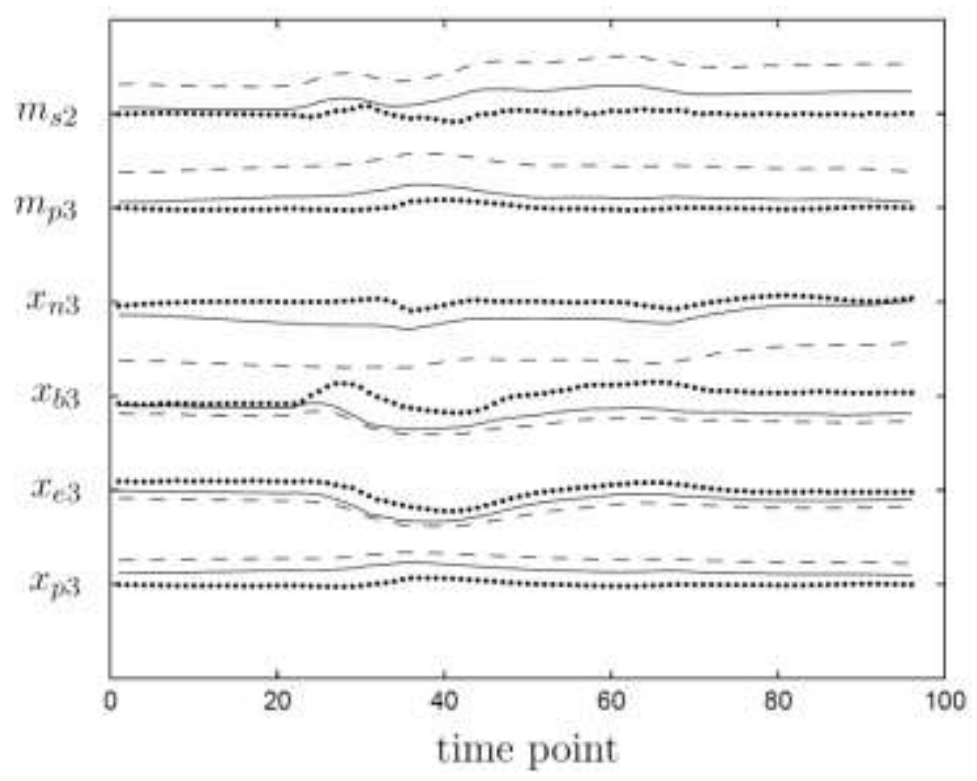

Figure 5. The calibrated model response (solid line) together with the measurement data (bold dots) and the nominal model response (dashed line) for dataset $T_{1}^{1}$.
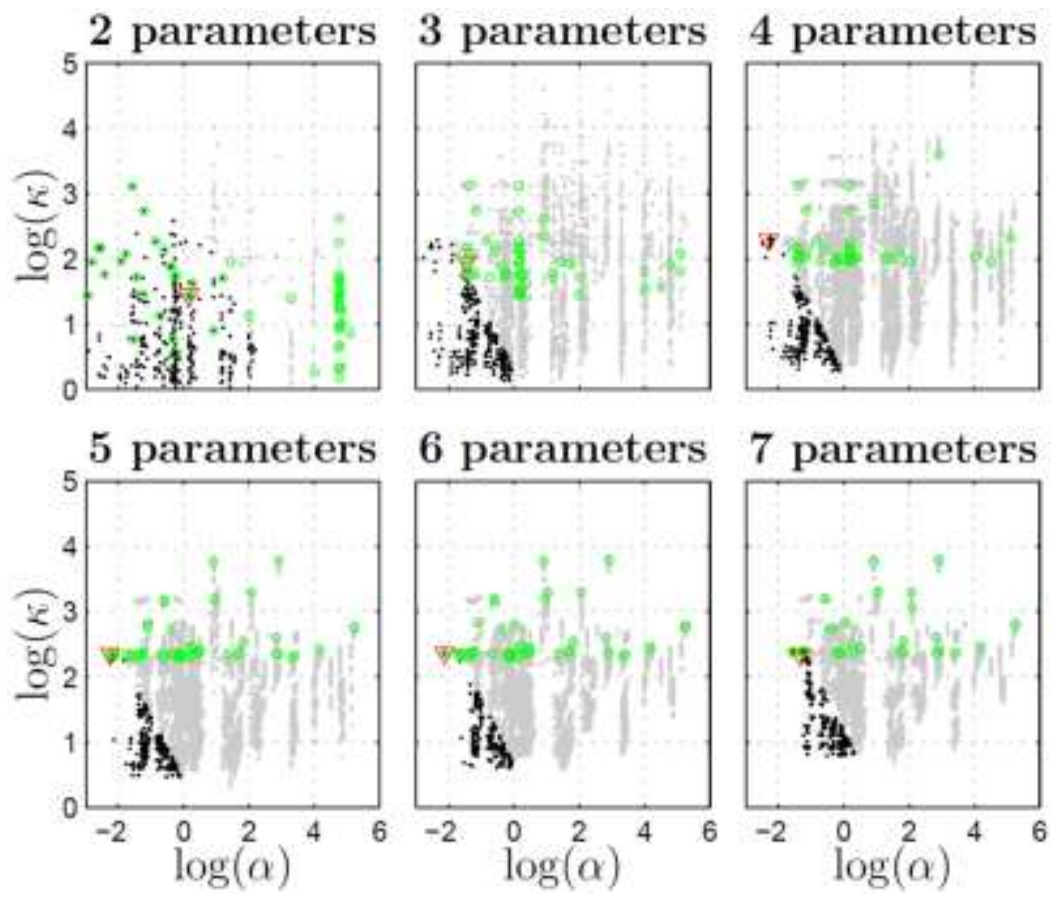

Figure 6. SSA evaluations for the dynamic case for two to seven number of parameters. The dots move to higher $\alpha$ and $\kappa$ values, as more parameters are included. The parameter sets are marked in gray, the best parameters from the SSA loop are marked in black and the parameters from the parameter estimation loop are marked in green. The parameter sets with the best objective values are marked with red triangles. 
Table 6. Results from the dynamic parameter estimation algorithm.

\begin{tabular}{|c|c|c|c|c|c|c|c|c|c|c|c|c|c|c|c|c|c|c|c|c|c|c|c|}
\hline$p_{V}^{2}$ & $p_{s 1}^{2}$ & $p_{s 3}^{2}$ & $p_{r e 1}^{2}$ & $p_{r h}^{2}$ & $p_{V}^{3}$ & $p$ & $\mathrm{x}$ & & & $\overline{p_{r e 3}^{3}}$ & & $\overline{p_{\text {re5 }}^{3}}$ & $\overline{p_{r b 1}^{3}}$ & $\overline{p_{r b 2}^{3}}$ & $\overline{p_{r b}^{3}}$ & $\overline{p_{o n}^{3}}$ & $\overline{p_{r e 2}^{A}}$ & $\overline{p_{r h 2}^{A}}$ & $p_{c 1}^{A}$ & $\overline{p_{c 2}^{A}}$ & $\frac{\log _{10} \alpha}{475}$ & $\frac{\log _{10} \kappa}{0}$ & $\begin{array}{r}\text { obj } \\
872\end{array}$ \\
\hline & & & & & & & & & & & & & & & & & & $\mathrm{x}$ & & & -2.98 & 0 & 87.8 \\
\hline & & & & & & & & & & & $\mathrm{x}$ & & & & & & & & & & 1.15 & 0 & 432 \\
\hline & & & & & & & & & $\mathrm{x}$ & & & & & & & & & & & & -0.30 & 0 & 432 \\
\hline & & & & & & & & & & & & $\mathrm{x}$ & & & & & & & & & 0.23 & 0 & 434 \\
\hline & & & & & & & & & & & & & & & $\mathrm{x}$ & & & $\mathrm{x}$ & & & 0.163 & 1.45 & 55.3 \\
\hline & & & & & & & & & & & & & $x$ & & & & & $x$ & & & -1.44 & 1.72 & 55.3 \\
\hline & & & & & & & & & & & & & & $\mathrm{x}$ & & & & $\mathrm{x}$ & & & 3.29 & 1.4 & 55.4 \\
\hline & & & & & & & & & & & & & & & & & & $x$ & & $x$ & -2.61 & 2.16 & 71.5 \\
\hline & & & & & & & & & & & & & & & & & & & $\mathrm{x}$ & & 1.99 & 1.13 & 72.9 \\
\hline & & & & & $\mathrm{x}$ & & & & & & & & $\mathrm{x}$ & & & & & $\mathrm{x}$ & & & $\begin{array}{l}-1.44 \\
\end{array}$ & 1.95 & 50.6 \\
\hline & & & & & $\mathrm{x}$ & & & & & & & & & & $\mathrm{x}$ & & & $\mathrm{x}$ & & & 0.163 & 1.95 & 50.6 \\
\hline & & & & & $\mathrm{x}$ & & & & & & & & & $\mathrm{x}$ & & & & $\mathrm{x}$ & & & 3.29 & 1.95 & 50.6 \\
\hline $\mathrm{x}$ & & & & & & & & & & & & & & & & & & $\mathrm{x}$ & & $x$ & -2.33 & 2.29 & 54.9 \\
\hline & & & & & $\mathrm{x}$ & & & & & & & & & & & & & $\mathrm{x}$ & & $x$ & -2.56 & 2.17 & 57.3 \\
\hline$x$ & & & & & $x$ & & & & & & & & & & & & & $x$ & & $x$ & -2.31 & 2.29 & 35.1 \\
\hline $\mathrm{x}$ & & & & & & & & & & & & & & & & & $\mathrm{x}$ & $x$ & & $\mathrm{x}$ & -2.26 & 2.33 & 42.8 \\
\hline & & & & & & & & & & $\mathrm{x}$ & & & $\mathrm{x}$ & & & $\mathrm{x}$ & & $\mathrm{x}$ & & & -1.12 & 1.82 & 51.1 \\
\hline & & & & & & & & & & $\mathrm{x}$ & & & & & $\mathrm{x}$ & $\mathrm{x}$ & & $\mathrm{x}$ & & & 0.178 & 1.55 & 51.1 \\
\hline & & & & & & & & & & $\mathrm{x}$ & & & & $\mathrm{x}$ & & $\mathrm{x}$ & & $\mathrm{x}$ & & & 3.3 & 1.53 & 51.1 \\
\hline $\mathrm{x}$ & & & & & $x$ & & & & & & & & & & & & $\mathrm{x}$ & $\mathrm{x}$ & & $x$ & -2.25 & 2.35 & 30.8 \\
\hline $\mathrm{x}$ & & & $\mathrm{x}$ & & $\mathrm{x}$ & & & & & & & & & & & & & $\mathrm{x}$ & & $x$ & -2.16 & 2.3 & 34.9 \\
\hline $\mathrm{x}$ & & & $\mathrm{x}$ & & & & & & & & & & & & & & $\mathrm{x}$ & $\mathrm{x}$ & & $\mathrm{x}$ & -2.13 & 2.34 & 42.5 \\
\hline & & & & $\mathrm{x}$ & & & & & & $\mathrm{x}$ & & & $x$ & & & $\mathrm{x}$ & & $\mathrm{x}$ & & & 1.46 & 2.07 & 48.8 \\
\hline & & & $\mathrm{x}$ & & $\mathrm{x}$ & & & & & & & & & & & & $\mathrm{x}$ & $\mathrm{x}$ & & $\mathrm{x}$ & -2.22 & 2.26 & 51.4 \\
\hline$x$ & & & $\mathrm{x}$ & & $x$ & & & & & & & & & & & & $x$ & $\mathrm{x}$ & & $x$ & -2.12 & 2.36 & 30.4 \\
\hline $\mathrm{x}$ & & & $\mathrm{x}$ & & $\mathrm{x}$ & & & $\mathrm{x}$ & & & & & & & & & & $\mathrm{x}$ & & $\mathrm{x}$ & -1.72 & 2.36 & 30.4 \\
\hline & $\mathrm{x}$ & & $\mathrm{x}$ & & $\mathrm{x}$ & & & & & & & & & & & & $\mathrm{x}$ & $\mathrm{x}$ & & $x$ & -0.71 & 2.41 & 30.5 \\
\hline & & $\mathrm{x}$ & $\mathrm{x}$ & & $\mathrm{x}$ & & & & & & & & & & & & $\mathrm{x}$ & $\mathrm{x}$ & & $\mathrm{x}$ & -0.45 & 2.34 & 30.5 \\
\hline & & & $\mathrm{x}$ & $\mathrm{x}$ & $\mathrm{x}$ & & & & & & & & & & & & $\mathrm{x}$ & $\mathrm{x}$ & & $x$ & 1.72 & 2.32 & 38 \\
\hline $\mathrm{x}$ & & & $x$ & & $\mathrm{x}$ & $\mathrm{x}$ & & & & & & & $\mathrm{x}$ & & & & & $\mathrm{x}$ & & $\mathrm{X}$ & -1.33 & 2.34 & 27.7 \\
\hline $\mathrm{x}$ & & & & & $\mathrm{x}$ & $x$ & & & & & & & $\mathrm{x}$ & & & & $\mathrm{x}$ & $\mathrm{x}$ & & $\mathrm{x}$ & -1.22 & 2.37 & 27.7 \\
\hline $\mathrm{x}$ & & & $\mathrm{x}$ & & $\mathrm{x}$ & & & & & & & & & & & $\mathrm{x}$ & $\mathrm{x}$ & $\mathrm{x}$ & & $x$ & -1.23 & 2.4 & 28.5 \\
\hline $\mathrm{x}$ & & & $\mathrm{x}$ & & $\mathrm{x}$ & & & $\mathrm{x}$ & & & & & $\mathrm{x}$ & & & & & $x$ & & $\mathrm{x}$ & -1.24 & 2.37 & 28.9 \\
\hline $\mathrm{x}$ & & & $\mathrm{x}$ & & $\mathrm{x}$ & & & & & & & & $\mathrm{x}$ & & & & $\mathrm{x}$ & $\mathrm{x}$ & & $\mathrm{x}$ & -1.28 & 2.37 & 28.9 \\
\hline 13 & 1 & 1 & 12 & 2 & 18 & 2 & 1 & 2 & 1 & 4 & 1 & 1 & 8 & 3 & 3 & 5 & 11 & 31 & 1 & 19 & & & \\
\hline
\end{tabular}

Table 7. Estimated objective values and cross validation for the best parameter set with five parameters for the dynamic case.

\begin{tabular}{ccccc}
\hline & $T_{1}^{1}$ & $T_{1}^{2}$ & $T_{2}^{1}$ & $T_{2}^{2}$ \\
\hline$p_{T_{1}^{1}}^{*}$ & 30.8 & 39.0 & 50.4 & 28.6 \\
$p_{T_{1}^{2}}^{*}$ & 40.0 & 30.4 & 29.8 & 19.1 \\
$p_{T_{2}^{1}}^{*}$ & 60.2 & 39.4 & 19.7 & 15.9 \\
$p_{T_{2}^{2}}^{*}$ & 68.3 & 49.4 & 34.3 & 10.6 \\
\hline$p_{\text {nom }}$ & 118.2 & 186.0 & 88.5 & 75.0 \\
\hline
\end{tabular}




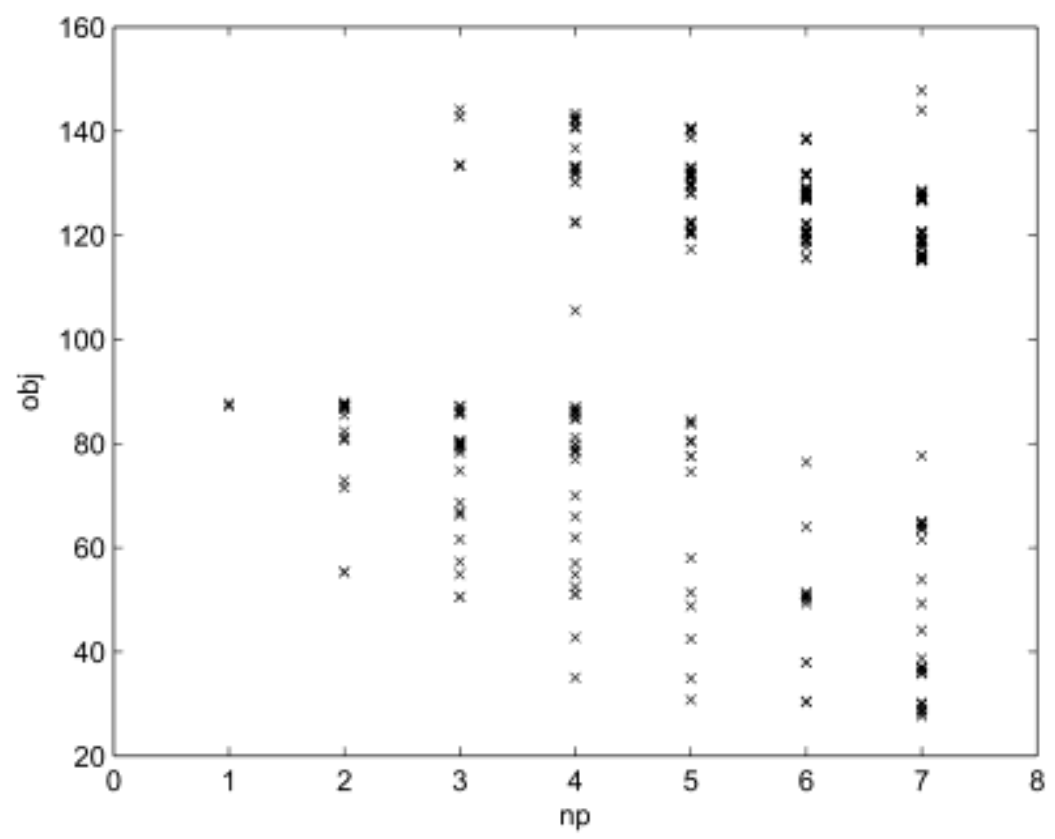

Figure 7. The objective values plotted against the number of parameters for dynamic parameter estimation

Table 8. Estimated scaled parameters for the different datasets for the best parameter set with five parameters for the dynamic case.

\begin{tabular}{lllccc}
\hline & \multicolumn{1}{c}{$p_{r e 1}^{A}$} & $p_{r e 2}^{A}$ & $p_{c 3}^{A}$ & $p_{V}^{2}$ & $p_{V}^{3}$ \\
\hline$p_{T_{1}^{1}}^{*}$ & 0.299 & 8.96 & 1.556 & 0.702 & 1.292 \\
$p_{T_{1}^{2}}^{*}$ & 0.0010 & 9.299 & 1.382 & 0.768 & 1.264 \\
$p_{T_{2}^{1}}^{*}$ & 0.0772 & 10.04 & 1.173 & 0.879 & 1.121 \\
$p_{T_{2}^{2}}^{*}$ & 0.417 & 10.13 & 1.149 & 0.791 & 1.216 \\
\hline
\end{tabular}

Table 6 shows that the best objective value for parameter sets with five parameters are $\left\{p_{r e 1}^{A}, p_{r e 2}^{A}, p_{c 3}^{A}, p_{V}^{2}, p_{V}^{3}\right\}$. This solution was optimised for the other datasets and Table 8 presents the parameter values obtained. The volume of the loop reactor is estimated lower in reality and that of the gas-phase reactor volume is estimated higher than in reality, for all parameter estimations. Two reaction rates for ethylene are included, where $p_{r e 1}^{A}$ varies between the datasets while $p_{r e 2}^{A}$ does not. The optimised values do not differ very much between the datasets, as in the steady-state case, except for parameter $p_{r e 1}^{A}$. This parameter set has a low $\alpha$ value and thus this result is expected.

Table 7 shows cross validations for the parameter estimations on the other datasets, where the optimal objective values from parameter estimations of the four datasets are located on the diagonal. Validations with the optimisation $T_{1}^{1}$ are shown on Row 1, and these agrees well with the grade transfer $T_{1}^{2}$, but somewhat poorer validations with $T_{2}^{1}$ and $T_{2}^{2}$. However, the objective values are much better than the objective values obtained with the nominal parameter values $p_{\text {nom }}$, and this is true for all validations. The validations are best within the same grade transfer for both $T_{1}$ and $T_{2}$. The validations between $T_{1}^{2}$ and $T_{2}^{1}$ are also very good, while the validations between $T_{1}^{1}$ and $T_{2}^{2}$ are not so good.

\subsection{Parameter Selection}

Table 4 and Table 6 show that many parameters can be directly eliminated from further analysis. In the steady-state case, 12 parameters $\left(p_{V}^{1}, p_{r h}^{1}, p_{r e 3}^{2}, p_{r h}^{2}, p_{r e 1}^{3}, p_{r e 3}^{3}, p_{r e 4}^{3}, p_{r e 5}^{3}, p_{o p}^{3}, p_{p c}^{3}, p_{r e 1}^{A}\right.$ and $\left.p_{c 1}^{A}\right)$ do not appear in the parameter sets that have the best objective values, and these can be discarded from further analysis. Similarly, in the dynamic case, 10 parameters $\left(p_{V}^{1}, p_{r e}^{1}, p_{r h}^{1}, p_{s 3}^{2}, p_{r e 2}^{2}, p_{r e 3}^{2}, p_{o p}^{2}, p_{p c}^{3}, p_{r e 1}^{A}\right.$ and $\left.p_{c 3}^{A}\right)$ do not appear in the parameter sets that have the best objective values, and these can be discarded. However, only six parameters $\left(p_{V}^{1}, p_{r h}^{1}, p_{r e 3}^{2}, p_{o p}^{3}, p_{p c}^{3}\right.$ and $\left.p_{r e 1}^{A}\right)$ occur neither in the steady-state nor in the dynamic case. Some parameters $\left(p_{V}^{2}, p_{s 1}^{2}, p_{s 3}^{2}, p_{r e 1}^{2}, p_{V}^{3}, p_{b e d}^{3}, p_{r h}^{3}, p_{r e 2}^{3}\right.$, $p_{r b 1}^{3}, p_{r b 2}^{3}, p_{r b 3}^{3}, p_{o n}^{3}, p_{r e 2}^{A}, p_{r h}^{A}$ and $\left.p_{c 2}^{A}\right)$ appear in both the steady-state case and the dynamic case.

Two parameters in the pre-polymerisation reactor $\left(p_{V}^{1}\right.$ and $\left.p_{r e}^{1}\right)$ do not appear in either case. This is due to the lack of measurements in the pre-polymerisation reactor, which leads to these parameters being less important. There were initially 11 kinetic parameters for ethylene: of these only $p_{r e 1}^{2}, p_{r e 2}^{3}$ and $p_{r e 2}^{A}$ appear in both cases. For the catalytic parameters, $p_{c 2}^{A}$ appears in both cases, $p_{c 1}^{A}$ only in the dynamic case and $p_{c 3}^{A}$ only in the steady-state case. 
The volumes that influence the residence times in the reactor are expected to be more important in the dynamic case than in the steady-state case. The results confirm this, in that $p_{V}^{2}$ and $p_{V}^{3}$ are found 13 and 18 times, respectively, in the dynamic case and only five and two times in the steady-state case. The volume of the pre-polymerisation reactor $p_{V}^{1}$ was not important in either cases, which may be explained by the lack of measurements.

\section{Summary and Conclusions}

This paper describes how to reduce the number of parameters in a model using an algorithm known as the subset selection algorithm. This algorithm ranks the parameters and reduces their number, and can be used to manage models with many potential parameter estimation parameters. We present results for a parameter reduction for a polyethylene producetion plant, in both steady-state and dynamic cases, and show that the two cases give different selections of parameters. The volume parameters were the most important parameters in the dynamic case, but not in the steady-state case.

We demonstrate that the objective value is not the only important attribute: numerical properties such as $\alpha$ and $\kappa$ are also important in obtaining good confidence intervals. The numerical properties become poorer as more parameters are added to the model. We show here that five parameters are sufficient to reach a plateau in the objective value in the model studied, in both the steady-state and the dynamic case. Adding further parameters does not improve the objective value and makes the system more numerically unstable. These effects leads to poorly calibrated parameters that are harder to validate to other datasets. It is also possible to select parameters by calibrating the process for each parameter individually and subsequently selecting the parameters that give the lowest objective values. This method, however, tends to give parameters that affect the system in similar manners, because several parameters are usually coupled to the objective function signals with the largest deviations. These parameters, such as $p_{r h}^{3}$ and $p_{r h}^{A}$ in the steady-state case, are often highly coupled and this coupling leads to poor numerical properties. This work demonstrates the disadvantages of the method and shows the benefits of the SSA method.

Parameters determined in the steady-state case were more difficult to validate with other datasets than parameters determined in the dynamic case. Parameter estimation of the system for different datasets gave significantly different parameter values, and high values of $\alpha$ and $\kappa$. The inflow parameters $u_{h 1}$ and $u_{h 3}$, which also were calibrated, probably played a major role in this effect. These variables were the main contribution to the high $\alpha$ and $\kappa$ values in the steady-state but were not included in the SSA analysis, where only parameters $\mathbf{p}$ were included. It would be possible to treat these parameters in the same way. Alternatively, omitting the two input parameters may give more reliable results for the steady-state case.

Validation of the dynamic case was successful, and all objective values lay below the objective values from the nominal parameter values. The parameter values were also calibrated in the same range for the parameters, which proves the model to be well-validated and of high quality. The method presented in this work requires only nominal parameter values and is thus easy to use. Further, the parameter Jacobian can be calculated at the start of the selection procedure.

\section{References}

Åkesson, J., Årzén, K. E., Gäfvert, M., Bergdahl, T., \& Tummescheit, H. ( 2010). Modeling and optimization with Optimica and JModelica.org - languages and tools for solving large-scale dynamic optimization problem. Computers and Chemical Engineering, 34(11), 1737-1749. http://dx.doi.org/10.1016/j.compchemeng.2009.11.011

Andersson, C., Andreasson, J., Führer, C., \& Åkesson, J. (2012). A workbench for multibody systems ode and dae solvers. In: 2nd Joint International Conference on Multibody System Dynamics. Stuttgart, Germany.

Andersson, N., Larsson, P., Åkesson, J., Haugwitz, S., \& Nilsson, B. (2011). Calibration of a polyethylene plant for grade change optimizations. In: 21st European Symposium on Computer Aided Process Engineering. Chalkidiki, Greece. http://dx.doi.org/10.1016/B978-0-444-53711-9.50135-8

Arora, N., \& Biegler, L. T. (2004). Parameter estimation for a polymerization reactor model with a composite-step trust-region nlp algorithm. Industrial \& Engineering Chemistry Research, 43(14), 3616-3631. http://dx.doi.org/10.1021/ie030237e

Bard, Y. ( 1970). Comparison of gradient methods for the solution of nonlinear parameter estimation problems. SIAM Journal on Numerical Analysis, 7 (1), 157-186. http://dx.doi.org/10.1137/0707011

Brun, R., Kühni, M., Siegrist, H., Gujer, W., \& Reichert, P. (2002). Practical identifiability of ASM2d parameters - systematic selection and tuning of parameter subsets. Water Research, 36(16), 4113-4127. http://dx.doi.org/10.1016/S0043-1354(02)00104-5

Cintrón, A., Banks, H. T., Capaldi, A., \& Lloyd, A. L. (2009). A Sensitivity Matrix Based Methodology for Inverse Problem Formulation. Journal of Inverse and Ill-Posed Problems, 17(6), 545-564. http://dx.doi.org/10.1515/JIIP.2009.034 
Edgar, T., \& Himmelblau, D. (1988). Optimization of Chemical Processes, 1st Edition. McGraw-Hill, New York, NY.

Englezos, P., \& Kalogerakis, N. (2000). Applied parameter estimation for chemical engineers, 1st Edition. CRC Press. http://dx.doi.org/10.1201/9780203904695

Faber, R., Li, P., \& Wozny, G. (2003). Sequential parameter estimation for large-scale systems with multiple data sets. 1. Computational framework. Industrial \& Engineering Chemistry Research, 42(23), 5850-5860. http://dx.doi.org/10.1021/ie030296s

Hindmarsh, A. C., Brown, P. N., Grant, K. E., Lee, S. L., Serban, R., Shumaker, D. E., \& Woodward, C. S. ( 2005). Sundials: Suite of nonlinear and differential/algebraic equation solvers. ACM Trans. Math. Softw., 31, 363-396. http://dx.doi.org/10.1145/1089014.1089020

Kravaris, C., Hahn, J., \& Chu, Y. (2013). Advances and selected recent developments in state and parameter estimation. Computers \& Chemical Engineering, 51, 111-123. http://dx.doi.org/10.1016/j.compchemeng.2012.06.001

Larsson, P. O. (2011). Optimization of low-level controllers and high-level polymer grade changes. Ph.D. thesis, Department of Automatic Control, Lund University, Sweden.

Larsson, P. O., Åkesson, J., Carlsson, N., \& Andersson, N. (2012a). Model-Based optimization of economical grade changes for the Borealis Borstar polyethylene plant. Computers \& Chemical Engineering, 46, 153-166. http://dx.doi.org/10.1016/j.compchemeng.2012.06.024

Larsson, P. O., Åkesson, J., Carlsson, N., \& Andersson, N. (2012b). Modeling of the PE3 Plant at Borealis AB. Tech. Rep. ISRN LUTFD2/TFRT--7622--SE, Dept. of Automatic Control, Lund University.

McLean, K. A., \& McAuley, K. B. (2012). Mathematical modelling of chemical processes—obtaining the best model predictions and parameter estimates using identifiability and estimability procedures. The Canadian Journal of Chemical Engineering, 90(2), 351-366. http://dx.doi.org/10.1002/cjce.20660

McAuley, K. B., MacGregor, J. F., \& McLellan, P. (1995). Effects of operating conditions on stability of gas-phase polyethylene reactors. AIChE Journal, 41(4), 868-879. http://dx.doi.org/10.1002/aic.690410414

Saarinen, M., \& Andersen, K. S. (2003). Applying Model Predictive Control in a Borstar Pilot Plant Polymerization Process, prepared for presentation at the session New Trends in Pilot Plants I, AIChE 2003 Spring National Meeting, New Orleans LA, March 31st.

Sirohi, A., \& Choi, K. Y. (1996). On-line parameter estimation in a continuous polymerization process. Industrial \& Engineering Chemistry Research, 35(4), 1332-1343. http://dx.doi.org/10.1021/ie950487y

The Modelica Association, 2011. The Modelica Association Home Page. http://www.modelica.org.

Vassiliadis, V. (1993). Computational solution of dynamic optimization problem with general differential-algebraic constraints. Ph.D. thesis, Imperial Collage, London, UK.

Weijers, S. R., \& Vanrolleghem, P. A. (1997). A procedure for selecting best identifiable parameters in calibrating activated sludge model no. 1 to full-scale plant data. Water Science and Technology, 36(5), 69-79. http://dx.doi.org/10.1016/S0273-1223(97)00463-0

Wächter, A., \& Biegler, L. T. (2006). On the implementation of an interior-point filter line-search algorithm for large-scale nonlinear programming. Mathematical Programming, 106(1), 25-58.

Zavala, V. M., \& Biegler, L. T. (2006). Large-scale parameter estimation in low-density polyethylene tubular reactors. Industrial and Engineering Chemistry Research, 45(23), 7867-7881. http://dx.doi.org/10.1007/s10107-004-0559-y

Zavala, V. M., \& Biegler, L. T. (2006). Large-scale parameter estimation in low-density polyethylene tubular reactors. Industrial and Engineering Chemistry Research, 45(23), 7867-7881. http://dx.doi.org/10.1021/ie060338n

Zhang, C., Shao, Z., Chen, X., Yao, Z., Gu, X., \& Biegler, L. T. (2014). Kinetic parameter estimation of HDPE slurry process from molecular weight distribution: Estimability analysis and multistep methodology. AIChE Journal, 60(10), 3442-3459. http://dx.doi.org/10.1002/aic.14527

\section{(cc) BY}

This work is licensed under a Creative Commons Attribution 3.0 License. 